\title{
El estado del arte de la comunicación de riesgos en la región de América Latina
}

\author{
Ana Rosa Moreno \\ (México, D.F., México) \\ Facultad de Medicina, Universidad Nacional Autónoma de México \\ Frederico Peres \\ (Río de Janeiro, Brasil) \\ Fundación Oswaldo Cruz
}

\section{Palabras clave}

comunicación riesgos

salud ambiental

América Latina

\begin{abstract}
Resumen
El cambio ambiental global y la diversidad de efectos en la salud humana han logrado en los últimos años que la sociedad en su conjunto esté atenta a lo que sucede en su entorno inmediato y mediato. Los organismos internacionales y los gobiernos reconocen la necesidad de evaluar y gestionar los riesgos para la salud y comunicarlos a todos los actores involucrados. Los principales esfuerzos en el tema de comunicación de riesgos en América Latina se han dado principalmente para enfrentar crisis, para el cuidado y, en menor medida, para el consenso. El tema de percepción de riesgos ha tenido un gran desarrollo en diversos países, principalmente por el sector académico. Conocer lo que la población opina o siente ante un peligro ambiental es fundamental para poder elaborar planes de comunicación de riesgos, estos son dos elementos estrechamente asociados. Actualmente y dados los retos en términos de las alteraciones ambientales que se prevén en el futuro cercano, la comunicación de riesgos debe ser un elemento de apoyo para fortalecer el conocimiento de las poblaciones en estos temas, así como promover que los tomadores de decisiones establezcan políticas públicas exitosas que protejan la salud de la ciudadanía.
\end{abstract}




\section{State of the Art of Risk \\ Communication in Latin America}

\begin{tabular}{c}
\hline Keywords \\
\hline risk communication \\
environmental health \\
Latin America
\end{tabular}

\section{Cómo citar el artículo}

Moreno, A. R. y Peres, F. (2011). El estado del arte de la comunicación de riesgos en la región de América latina. Revista de Comunicación y Salud, 1(1), pp. 55-71.

DOI: http://doi.org/10.35669/revistadecomunicacionysalud.2011.1(1).55-71

\section{Antecedentes}

El cambio ambiental global manifestado por el daño en la capa de ozono, el cambio climático, cambios del uso del suelo y su degradación, disminución de fuentes de agua potable y su contaminación, así como la pérdida de la biodiversidad son procesos que afectan la salud humana a través de diversos mecanismos. Por ejemplo, la contaminación afecta especialmente a la población infantil, por hallarse en desarrollo orgánico y neuropsicológico.

Cada día la sociedad es más consciente de estos cambios ambientales a través de lo observado en su cotidianeidad y lo difundido por los medios de comunicación, de tal manera que los gobiernos se ven en la necesidad de controlar los riesgos derivados de la industrialización, el desarrollo tecnológico y el crecimiento no planificado de las grandes ciudades. 
Se entiende como salud ambiental aquella área del conocimiento en que confluyen el medio ambiente y la salud para conocer aquellas situaciones que pueden representar un riesgo para la salud individual y colectiva, al exponerse a las modificaciones del medio ambiente donde las personas viven y trabajan, con el fin de promover políticas públicas, tanto en el campo ambiental como de salud.

A partir de evaluaciones hechas en los últimos años por la Organización Mundial de la Salud respecto a los efectos del medio ambiente en la salud se ha llegado a estimar que los factores de riesgo ambiental tienen, a nivel mundial, un rol significativo en más de 80 importantes enfermedades y lesiones. El 24 por ciento de la carga de morbilidad (años de vida sana perdidos) y el 23 por ciento de las muertes (mortalidad prematura) han sido atribuidas a factores ambientales, por lo que podrían haberse prevenido mediante intervenciones en el medio ambiente. En el caso de niños entre 0 y 14 años la cifra llega hasta un 36 por ciento. Cuando se analiza las regiones del mundo se reporta que el 25 por ciento de todas las muertes registradas en países en desarrollo se atribuía a causas ambientales, a diferencia de los países desarrollados en donde solo al 17 por ciento se le atribuían estas causas (Prüss-Üstün y Corvalán, 2006).

Al tener estas evidencias del componente ambiental en los patrones de salud y enfermedad en una población se hace evidente la necesidad de proporcionar información a todos los sectores que pueden verse afectados por la exposición a agentes ambientales peligrosos. De aquí se deriva, a su vez, la necesidad de comunicar los riesgos a las diversas poblaciones involucradas.

\section{La comunicación de riesgos como una herramienta en salud ambiental}

La comunicación de riesgos ha sido definida como un proceso interactivo de intercambio de información y de opiniones entre individuos, grupos e instituciones. Es un diálogo en el cual se discuten múltiples mensajes que expresan preocupaciones, opiniones o reacciones a los propios mensajes o arreglos legales e institucionales del manejo de riesgos (National Research Council, 1989). Es un intercambio de información a propósito en la interacción entre personas involucradas con respecto a salud, seguridad y amenazas ambientales. Abarca cualquier comunicación que informe a los individuos acerca de la existencia, naturaleza, forma, severidad y aceptabilidad de un riesgo.

Uno de los temas en donde la comunicación de riesgos se vuelve un elemento fundamental es en los sistemas de alerta temprana ante eventos extremos como huracanes u olas de calor. Por ejemplo, solamente en el año 2005, los huracanes dejaron un saldo de 4.598 muertos en América Latina (OPS, 2007), así que la comunicación de riesgos proporciona la forma de acercarse a los grupos vulnerables con el fin de proteger la salud y disminuir la morbilidad y la mortalidad.

Las iniciativas de comunicación de riesgo deben diseñarse de tal manera que se asegure que los mensajes lleguen a los grupos objetivo de la población, de la forma más clara y sucinta, en tiempo preciso y a través del canal pertinente para la audiencia a la que van dirigidas. Para ello, primero se deben encontrar formas de desagregar las diferencias y necesidades 
individuales e incluir en la información que se proporcione las preocupaciones sentidas por el público (OPS, 2007).

En la tabla 1 se señalan los diferentes roles organizacionales que se pueden identificar en la comunicación de riesgos, cada uno de ellos tendrá su propia sus propias expectativas y autoridad responsable.

Tabla 1. Ejemplo de roles organizacionales en la comunicación de riesgos

\begin{tabular}{|l|l|l|}
\hline Papel & Expectativa & Autoridad \\
\hline Educador & Explicar, informar & Impartir conocimiento \\
\hline Facilitador & Animar, apoyar & Señalar soluciones \\
\hline Socio & Trabajar con otros & Solucionar problemas \\
\hline Administrador & Decir qué hacer & Prevenir o mitigar el riesgo \\
\hline Regulador & Adjudicar justicia & Hacer cumplir las decisiones \\
\hline
\end{tabular}

Fuente: Lundren y McMakin, 2004: 51.

Desde la década de los 70 se han visto grandes controversias públicas sobre la gestión de riesgos sanitarios y ambientales que han sido constantes, desde el uso masivo de productos químicos, el riesgo asociado con muchas otras tecnologías industriales, hasta el cambio climático. Esta conciencia ciudadana ha conducido a que haya un mayor interés por los daños ambientales y sus consecuentes efectos para la salud de la gente, trayendo con ello las diversas controversias que se basan en la discusión de los posibles efectos debidos a la exposición a diversos agentes químicos y físicos, así como los consecuentes riesgos para las poblaciones humanas, incluyendo aquellos individuos que pudieran ser más vulnerables.

Como resultado de lo anterior, en los últimos años América Latina se ha interesado por dar a conocer información a diferentes sectores de la sociedad con el fin de responder de forma adecuada a los riesgos a los que los diversos grupos etarios se enfrentan y poder prevenir efectos a corto o largo plazo; sin embargo, después de la etapa de informar, cada día se tiene mayor conciencia de la necesidad de comunicar, con lo que la población deja de ser pasiva como receptora a ser activa al involucrarse con aquellos responsables del riesgo.

La evaluación y gestión de dichos riesgos, metodologías que permiten conocer los peligros físicos y químicos a los que se enfrenta la población y cómo manejarlos, se apoyan en la comunicación de riesgos. Además, la comprensión de los problemas de salud relacionados con el medio ambiente permite proponer estrategias a las organizaciones e instituciones que les ayuden a responder mejor a las necesidades del público ( $\mathrm{y}$ a su interés) en cuanto a las preocupaciones que tiene sobre los diversos riesgos. Prácticas eficaces de comunicación de riesgos se encuentran entre las responsabilidades más importantes para la industria y los gobiernos en este sentido (Leiss, 2004). Los gobiernos enfrentan el reto de mantener una distinción clara entre las técnicas de comunicación vistas por el público como propaganda y aquellas diseñadas para proporcionar información técnica, promover, educar y cambiar actitudes. Esto implica otro problema al asumir el gobierno el rol de comunicador y de regulador. 
Lo anterior ha conducido a que los gobiernos establezcan políticas que permitan el acceso a la información por parte del público, de grupos activistas y de los medios respecto de problemas ambientales y de salud. Sin embargo, este esfuerzo no acaba de plasmar en los países en vías de desarrollo, lo que ocasiona muchas veces conflictos innecesarios e inquietudes por la falta de información y por los obstáculos en la participación ciudadana (Moreno et al., 2010). Estos problemas se presentan ya que, como se ha mencionado, la comunicación implica una relación en donde se reciben y discuten mensajes con la audiencia, lo que permite la participación de los ciudadanos; ofrecer información no garantiza este involucramiento y/o cambios de conducta. Un proceso de comunicación ordenado propicia una relación permanente con la población interesada o afectada con base en el respeto, confianza, credibilidad y profesionalismo que lleve a alcanzar un bien común.

La figura 1 señala la posición central que tiene la comunicación de riesgos en los procesos de evaluación y gestión, y desarrollo, instrumentación y evaluación de políticas en el sector de medio ambiente y salud.

Figura 1. Ciclo del manejo del riesgo

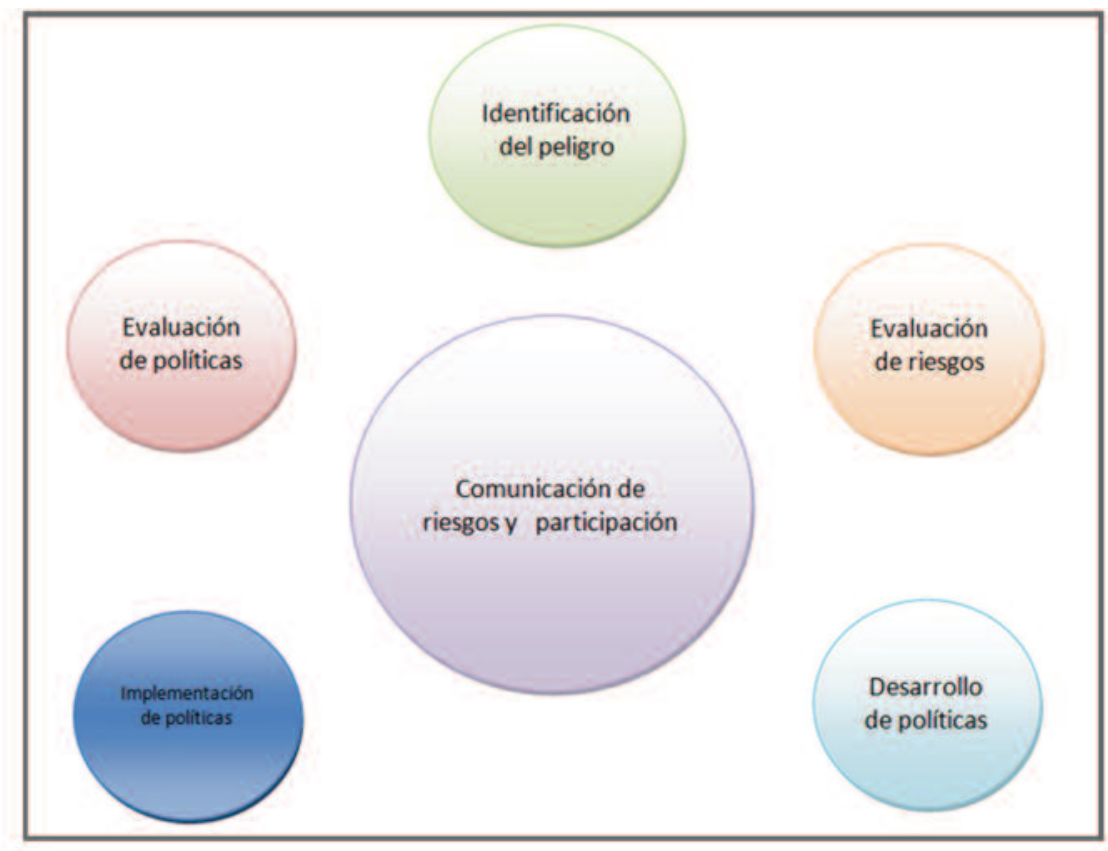

Fuente: Lang et al. (2001).

Algunas de las diversas experiencias reportadas en comunicación de riesgos en la región de América Latina se muestran en la tabla 2, a partir de la cual se identifican dos ejes principales de acción. El primero y más importante es la comunicación de riesgos ante desastres, experiencia bien planificada y evaluada para prácticamente todos los países de la región. Esta experiencia ha tenido un impacto positivo muy importante tanto en la morbilidad como en la mortalidad, además ha pasado a ser de gran importancia a partir de los escenarios de cambio 
climático que señalan el aumento e intensidad en fenómenos hidrometeorológicos. El siguiente eje es el propuesto por los planes de respuesta ante la pandemia de influenza en donde la comunicación se plantea bien diseñada y estructurada, siendo una iniciativa liderada por la OPS; a partir de la aparición de la gripe H1N1 este tipo de capacitación se ha visto incrementada con la necesidad de contar con una respuesta en tiempo y forma de todos los sectores de la sociedad. Esta Organización trabaja con todos los países en la Región para crear estrategias de comunicación que se puedan implementar de una manera intersectorial, destacando la necesidad de informar, educar, comunicar y abogar (OPS, 2007).

Se ha hecho una búsqueda en la web con el fin de identificar evaluaciones para planes diferentes a los de desastres, sin embargo no se encontró ningún reporte de evaluación de otros esfuerzos de comunicación de riesgos; posiblemente si se llegan a hacer no se publican los resultados o bien puede quedar como un ejercicio académico difícil de detectar. Esto impide el aprendizaje de dichas experiencias y la optimización de los planes.

Tabla 2. diversas actividades de comunicación de riesgos en América latina

\begin{tabular}{|c|c|}
\hline País & Actividades \\
\hline \multirow{4}{*}{ Argentina } & Participación en la Red de Estudios Sociales en Prevención de Desastres en América Latina \\
\hline & Capacitación* \\
\hline & Inocuidad de alimentos \\
\hline & Planes de respuesta ante la pandemia de influenza \\
\hline Belice & Planes de respuesta ante la pandemia de influenza \\
\hline \multirow{4}{*}{ Brasil } & Participación en la Red de Estudios Sociales en Prevención de Desastres en América Latina \\
\hline & Inocuidad de alimentos \\
\hline & Planes de respuesta ante la pandemia de influenza \\
\hline & Capacitación* \\
\hline \multirow{4}{*}{ Bolivia } & Inocuidad de alimentos \\
\hline & Prevención de desastres \\
\hline & Planes de respuesta ante la pandemia de influenza \\
\hline & Capacitación* \\
\hline \multirow{3}{*}{ Costa Rica } & Participación en la Red de Estudios Sociales en Prevención de Desastres en América Latina \\
\hline & Planes de respuesta ante la pandemia de influenza \\
\hline & Capacitación* \\
\hline \multirow{4}{*}{ Colombia } & Participación en la Red de Estudios Sociales en Prevención de Desastres en América Latina \\
\hline & Inocuidad de alimentos \\
\hline & Planes de respuesta ante la pandemia de influenza \\
\hline & Capacitación* \\
\hline
\end{tabular}

\footnotetext{
* En algunos casos es comunicación de riesgos en general y es promovida por empresas privadas, por universidades como cursos de educación continua, de un programa de maestría o bien dirigidos a personal de organismos oficiales. En otros casos, es capacitación en comunicación de riesgos ante brotes.
} 


\begin{tabular}{|c|c|}
\hline País & Actividades \\
\hline \multirow{3}{*}{ Chile } & Inocuidad de alimentos \\
\hline & Planes de respuesta ante la pandemia de influenza \\
\hline & Capacitación* \\
\hline \multirow{4}{*}{ Ecuador } & Participación en la Red de Estudios Sociales en Prevención de Desastres en América Latina \\
\hline & Inocuidad de alimentos \\
\hline & Planes de respuesta ante la pandemia de influenza \\
\hline & Capacitación* \\
\hline \multirow{3}{*}{ El Salvador } & Participación en la Red de Estudios Sociales en Prevención de Desastres en América Latina \\
\hline & Capacitación* \\
\hline & Planes de respuesta ante la pandemia de influenza \\
\hline \multirow{2}{*}{ Guatemala } & Participación en la Red de Estudios Sociales en Prevención de Desastres en América Latina \\
\hline & Capacitación ${ }^{*}$ \\
\hline Honduras & Capacitación* \\
\hline \multirow{6}{*}{ México } & $\begin{array}{l}\text { Normatividad para contar con un sistema para la identificación y comunicación de riesgos por } \\
\text { sustancias químicas en los centros de trabajo }\end{array}$ \\
\hline & $\begin{array}{l}\text { Normatividad para el Distrito Federal que establece los requisitos para elaborar el Índice Metro- } \\
\text { politano de la Calidad del Aire }\end{array}$ \\
\hline & Participación en la Red de Estudios Sociales en Prevención de Desastres en América Latina \\
\hline & $\begin{array}{l}\text { Acciones de comunicación de riesgos por parte de la Comisión para la Protección de Riesgos Sa- } \\
\text { nitarios, dependencia del sector salud a nivel federal }\end{array}$ \\
\hline & Planes de respuesta ante la pandemia de influenza \\
\hline & Capacitación* \\
\hline Nicaragua & Capacitación* \\
\hline \multirow{3}{*}{ Panamá } & Capacitación* \\
\hline & Planes de respuesta ante la pandemia de influenza \\
\hline & Planes de comunicación de riesgos ante la exposición a radiaciones y mercurio \\
\hline \multirow{4}{*}{ Paraguay } & Inocuidad de alimentos \\
\hline & Planes de respuesta ante la pandemia de influenza \\
\hline & Prevención de desastres \\
\hline & Capacitación* \\
\hline \multirow{5}{*}{ Perú } & Participación en la Red de Estudios Sociales en Prevención de Desastres en América Latina \\
\hline & Inocuidad de alimentos \\
\hline & Prevención de desastres \\
\hline & Planes de respuesta ante la pandemia de influenza \\
\hline & Capacitación* \\
\hline \multirow{4}{*}{ Uruguay } & Inocuidad de alimentos \\
\hline & Planes de respuesta ante la pandemia de influenza \\
\hline & Prevención de desastres \\
\hline & Capacitación* \\
\hline \multirow{2}{*}{ Venezuela } & Participación en la Red de Estudios Sociales en Prevención de Desastres en América Latina \\
\hline & Planes de respuesta ante la pandemia de influenza \\
\hline
\end{tabular}

Fuente: elaboración propia 
Se puede señalar también que ha habido una serie de experiencias de capacitación en la región, algunas veces a partir de un eje temático (p. ej. plaguicidas) y en algunas otras sólo para dar a conocer el tema y la metodología.

La comunicación eficaz aclara concepciones erróneas ayudando a que la gente tome decisiones basadas en información completa y específica. Cuando se le da la oportunidad a la ciudadanía para participar activamente en discusiones enfocadas en minimizar y controlar riesgos de salud se abre la oportunidad para que se minimice el resentimiento de las personas que se sienten excluidas de las políticas de salud y propicia que se mantengan las normas o se cambie el statu quo, logrando reducir la morbilidad, salvar vidas y empoderar a las personas.

\section{La producción científica sobre comunicación de riesgos en América Latina}

Al analizar la producción científica sobre comunicación de riesgos entre los grupos de investigación en América Latina (tabla 3), es posible visualizar que representa una limitada contribución en comparación con América del Norte y las producciones europeas. Sin embargo, es importante destacar que estas contribuciones van en aumento, particularmente en Brasil y México, que muestran el potencial de los nuevos grupos de investigación en la Región.

Tabla 3. Artículos latinoamericanos en comunicación de riesgos

(Archivos en Medline/PubMed de 2000 a 2010). De 2000 a 2010, se publicaron y referenciaron 945 documentos en PubMed como comunicación de riesgos, nueve de ellos fueron producidos por grupos de investigación latinoamericanos.

\begin{tabular}{|l|c|c|c|}
\hline \multicolumn{1}{|c|}{ Autor(es) } & Año & Objetivo de la Comunicación de Riesgo & País \\
\hline Trasher et al. & 2010 & Legislación anti-tabaco en la Cd. De México & México \\
\hline Echavarría-Zuno et al. & 2009 & Epidemia de gripe H1N1 en México & México \\
\hline Córdova-Villalobos et al. & 2009 & Epidemia de gripe H1N1 en México & México \\
\hline Rangel & 2007 & Revisión de comunicación de riesgo & Brasil \\
\hline Peres et al. & 2006 & Exposición a plaguicidas en el trabajo rural & Brasil \\
\hline Kuri-Morales et al. & 2006 & Pandemia de la influenza & México \\
\hline Peres et al. & 2005 & Exposición a plaguicidas en el trabajo rural & Brasil \\
\hline Peres et al. & 2001 & Exposición a plaguicidas en el trabajo rural & Brasil \\
\hline Boischio \& Henshel & 2000 & Contaminación con mercurio en peces & Brasil \\
\hline
\end{tabular}

Fuente: modificado de Moreno et al., 2010.

La mayoría de la producción científica de la comunicación de los riesgos en América Latina se asocia con los estudios de percepción del riesgo, esto muestra una posible tendencia y pone 
en evidencia la aplicación de la comunicación de riesgos para el cuidado, y menos para la atención de crisis o para consenso.

\subsection{Percepción del riesgo y comunicación del riesgo: dimensiones no disociables.}

Desde la década de 1970, cuando el mundo comenzó a enfrentar la compleja dimensión de los impactos en la salud ambiental por proyectos tecnológicos, se hizo evidente que para tener una mejor comprensión y gestión de los riesgos relacionados con la exposición humana a contaminantes -así como los derivados de los diferentes procesos de trabajo- y, por tanto, la elaboración de estrategias de comunicación, era necesario considerar el contexto social en el que los riesgos se produjeron, como determinantes de la vulnerabilidad en muchos grupos de población específicos. El análisis de estos aspectos es, en nuestra realidad, tan importante como la identificación y el análisis de los riesgos de las tecnologías, procesos de trabajo y los contaminantes del medio ambiente en sí mismos.

El análisis de los riesgos sociales, considerando el contexto social y su dinámica, nos permite no sólo el conocimiento de la realidad, sino también la construcción de propuestas enfocadas en su control y prevención, y con esto se sientan las bases del problema en el corto, mediano y largo plazos. El reto que se plantea, en este caso, es la posibilidad de incorporación de conocimientos, creencias y aspiraciones de las comunidades en el análisis de riesgos y la gestión, así como de información sobre la interrelación de la subjetividad individual y el orden social, la experiencia práctica e imaginaria, y los datos cuantitativos y cualitativos (Rozemberg y Peres, 2003).

En esto contexto, los estudios de percepción de riesgo surgieron, particularmente en los EEUU y en algunos países europeos, como una alternativa al análisis de riesgos utilitario y técnico basado en ingeniería, toxicología, economía y ciencias actuariales que ignoran las creencias, temores y dudas de las comunidades involucradas. Estos estudios se hicieron más sólidos y se convirtieron en un campo científico organizado con el propósito de entender las reacciones negativas de los legos (o no especialistas) en las nuevas tecnologías.

Una definición de la percepción del riesgo es "la capacidad que tiene un persona para interpretar una situación potencialmente dañina para la salud o la vida propia o de otros, con base en experiencias previas y en proyecciones futuras, que pueden variar de una opinión vaga a una firme convicción" (Wiedemann, 1993: 3). El autor propone, a raíz de esta definición, que la percepción del riesgo se basa principalmente en las concepciones y creencias, y en menor medida, en experiencias personales previas.

De acuerdo con Moreno et al. (2010), existen tres elementos fundamentales para entender esta proximidad entre la percepción y comunicación de riesgos. En primer lugar, la comunicación de riesgos es un diálogo, un proceso de dos vías, la comunicación va más allá de proveer información, sino que es el resultado de las interacciones entre todos los actores involucrados en el proceso de toma de decisiones. Al mismo tiempo, los técnicos (expertos) y la comunidad (no-expertos) son los informantes (emisores) y los receptores de toda la información, en un proceso dialógico que es atravesado verticalmente por elementos multidimensionales, lo que podría 
ser a la vez relacionado con los aspectos técnicos del proceso de comunicación (p.ej. material, contenido técnico, el instrumento elegido, los medios de comunicación, etc.) y/o factores socioculturales (p.ej. la cultura, el curso de vida, patrones de lenguaje, etc.). Todo ello se puede ver en la figura 2.

En segundo lugar, la comunicación de riesgos es también la educación, y ésta es la transformación a través del conocimiento. Esto significa que si el profesional no entiende el universo en el que trabaja, tiende a transmitir el conocimiento producido en su realidad específica (académica) para un grupo de población distinta, promoviendo no sólo una falla en la práctica educativa, sino también una ruptura y tergiversación de las normas culturales locales/tradicionales.

Figura 2. Proceso dialógico y las dimensiones de comunicación de riesgos

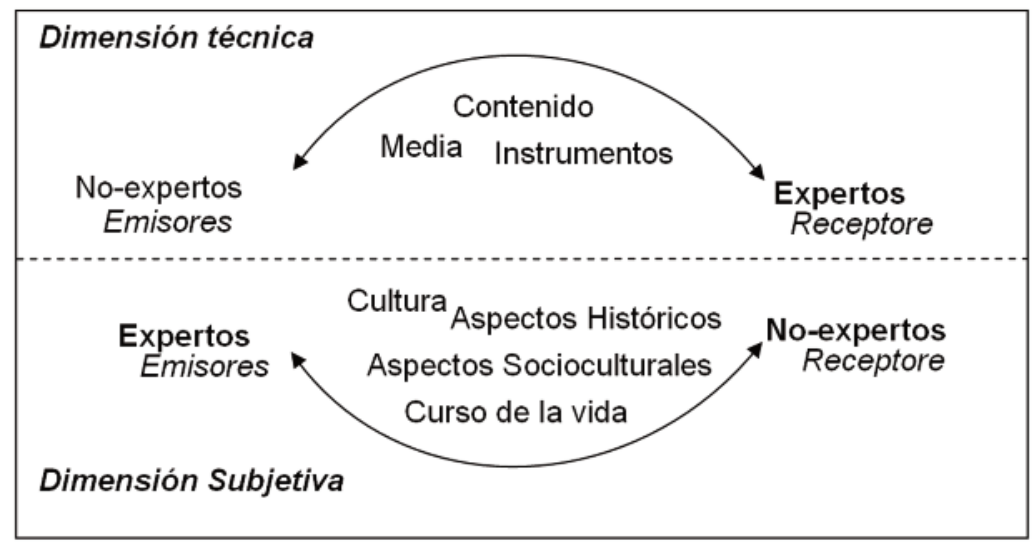

Fuente: elaboración propia

Por último, la comunicación de riesgos depende de la confianza y credibilidad. Estas condiciones son construidas colectivamente y de forma permanente y, por lo tanto, su atención en una estrategia de comunicación de riesgos es permanente. Toda la comunicación es totalmente dependiente de la confianza que cada actor tiene hacia otro. En un proceso de comunicación de riesgos, que a menudo involucra conflicto de intereses, este aspecto es más evidente que nunca.

Podríamos decir, de acuerdo con Moreno et al. (2010), que la implementación exitosa de estrategias de intervención frente a los complejos problemas de salud ambiental, tales como la exposición a plaguicidas, la intoxicación por plomo, la contaminación ambiental y otros problemas relacionados con el cambio climático, por ejemplo, significará un desafío interdisciplinario que expone a los profesionales de muchas disciplinas a los principios básicos y las contribuciones de cada campo y apuntan a la necesidad de que cada profesional entienda la ciencia, los métodos, la preparación y el jerga del otro.

Es necesario dar más importancia a las creencias de las comunidades, incluso si se consideran insignificantes en un primer vistazo. También es necesario comprender que la distancia entre los especialistas y no especialistas, es la misma en ambos sentidos: mientras los especialistas 
están lejos de la realidad de las comunidades, de la misma manera estarán lejos de la comprensión real de sus problemas. Lo que se percibe como real por la población, ya sea correcto o no, es real para las personas y también en sus consecuencias. Estas percepciones de riesgo deben ser tomadas en consideración al formular una iniciativa de comunicación de riesgos, ya sea como punto de partida para la construcción de una nueva idea (que incluso puede oponerse), o ya sea como un elemento que podría cambiar el enfoque y permitir ajustes en la planificación de la comunicación de riesgos.

En la Región, los estudios de percepción del riesgo surgieron después de la segunda mitad de la década de 1990, en particular relacionados con el riesgo de infección por $\mathrm{VIH} / \mathrm{sida}$ (se pueden encontrar algunos estudios a principios de dicha década sobre este mismo tema). Estas iniciativas se incrementaron de forma significativa después de 2000, mostrando el potencial de adaptar estrategias de percepción del riesgo en América Latina (tabla 4), en particular en los enfoques de evaluación y gestión de riesgos. Aunque la mayoría de ellos todavía reportan resultados de proyectos de investigación sobre enfermedades de transmisión sexual (ETS), $\mathrm{VIH} /$ sida o riesgos ocupacionales, las investigaciones en salud ambiental son evidentes, sobre todo en la producción de los últimos cinco años, donde las estrategias de percepción del riesgo toman una posición central para conectar la investigación con la acción (Moreno et al., 2010).

Tabla 4. Artículos latinoamericanos en percepción del riesgo.

(Archivos de Medline/PubMed de 2000 a 2010). De 2000 a 2010, se publicaron y referenciaron 1.020 artículos en PubMed como comunicación de riesgos, 41 fueron producidos por grupos de investigación latinoamericanos

\begin{tabular}{|c|c|c|c|}
\hline Autor(es) & Año & Objetivo de la percepción de riesgo & País \\
\hline Melo et al. & 2010 & Infección por VIH / SIDA & Brasil \\
\hline Catalán-Vasquez et al. & 2010 & Exposición a manganeso por actividades de la minería & México \\
\hline Lopez-Quintero et al. & 2010 & Uso de drogas en adolescentes & Colombia \\
\hline Cezar-Vaz et al. & 2009 & Riesgos ocupacionales en profesionales de la salud & Brasil \\
\hline Neves \& Melo & 2009 & Riesgos ocupacionales en la milicia & Brasil \\
\hline Dinelli et al. & 2009 & Riesgos ocupacionales en profesionales de la salud & Brasil \\
\hline Nathan F. & 2008 & Accidentes en residentes que habitan en zonas con pendientes & Bolivia \\
\hline Katagiri S. et al. & 2008 & Zoonosis entre dueños de perros & Brasil \\
\hline Gil HL et al. & 2008 & Consumo de drogas en estudiantes & Perú \\
\hline Ferreira MP & 2008 & Infección por VIH / SIDA & Brasil \\
\hline Recena MC et al. & 2008 & Exposición a plaguicidas en el trabajo rural & Brasil \\
\hline Thielen IP et al. & 2008 & Exceso de velocidad / Accidentes de coche & Brasil \\
\hline Trujillo AM et al. & 2007 & Uso (abuso) de sustancias en jóvenes & Colombia \\
\hline Kendall et al. & 2007 & Infección por VIH / SIDA & México \\
\hline Fonseca et al. & 2007 & Exposición a plaguicidas en el trabajo rural & Brasil \\
\hline
\end{tabular}




\begin{tabular}{|c|c|c|c|}
\hline Autor(es) & Año & Objetivo de la percepción de riesgo & País \\
\hline Bird $\mathrm{Y}$ et al. & 2007 & Exposición estudiantil al tabaco / tabaquismo & México \\
\hline Castillo-Arriaga A et al. & 2007 & Diabetes & México \\
\hline Peres F et al. & 2007 & Exposición a plaguicidas en el trabajo rural & Brasil \\
\hline Salazar X et al. & 2007 & VIH / AIDS infection & Perú \\
\hline Maia $\mathrm{H}$ et al. & 2006 & Terapia hormonal en la menopausia & Brasil \\
\hline Peres F et al. & 2005 & Exposición a plaguicidas en el trabajo rural & Brasil \\
\hline Baltazar Reyes MC et al. & 2005 & Prevalencia de enfermedades de transmisión sexual & México \\
\hline Filipe EM et al. & 2005 & Infección por VIH / SIDA & Brasil \\
\hline Peres et al. & 2004 & Exposición a plaguicidas en el trabajo rural & Brasil \\
\hline Dias MA et al. & 2004 & Cesáreas & Brasil \\
\hline Ferreira MP & 2003 & Infección por VIH / SIDA & Brasil \\
\hline Tate RB et al. & 2003 & Promoción para la salud & Cuba \\
\hline Bronfman NC et al. & 2003 & Peligros ambientales para la salud & Chile \\
\hline Praça NS et al. & 2003 & Infección por VIH / SIDA & Brasil \\
\hline Segurado AC & 2003 & Infección por VIH / SIDA & Brasil \\
\hline Peres CA et al. & 2003 & Infección por VIH / SIDA & Brasil \\
\hline Antunes MC et al. & 2002 & Infección por VIH / SIDA & Brasil \\
\hline Alves RN et al. & 2002 & Infección por VIH / SIDA & Brasil \\
\hline Tunala LG & 2002 & Infección por VIH / SIDA & Brasil \\
\hline Santos NJ et al. & 2002 & Infección por VIH / SIDA & Brasil \\
\hline Iriart JA et al. & 2002 & Uso de esteroides y fisicoculturismo & Brasil \\
\hline Cordeiro R & 2002 & Lesiones laborales & Brasil \\
\hline Castor D & 2002 & Infección por gonorrea & Trinidad \\
\hline Vieira EM et al. & 2001 & Conducta sexual y enfermedades de transmisión sexual & Brasil \\
\hline Ventura-Filipe EM et al. & 2000 & Infección por VIH / SIDA & Brasil \\
\hline Fernandez $\mathrm{N}$ et al. & 2000 & Promoción para la salud & Cuba \\
\hline
\end{tabular}

Fuente: modificado de Moreno et al., 2010.

De acuerdo con Moreno et al. (2010), Brasil es el país responsable de la mayoría de los estudios latinoamericanos en percepción del riesgo, en particular los relacionados con el VIH/sida. Recientemente, muchos estudios comenzaron a reportar iniciativas de percepción del riesgo asociadas con estudios de salud ambiental, demostrando su uso potencial para desarrollar una mejor comprensión de cómo los grupos vulnerables enfrentan los riesgos a los que están expuestos.

A partir de diferentes enfoques se ha conducido una discusión sobre la dicotomía de la percepción entre los especialistas y no especialistas en varios grupos académicos desde 1970 (Brown, 1992; Kleinman, 1980; Kleinman y Good, 1986; Sjöberg y Fromm, 2001; Zola, 1973). A pesar de las controversias relacionadas con estos debates, son importantes para comprender los conflictos de interés en la gestión de problemas de salud ambiental, así como las dimensiones subjetivas co-relacionadas. Uno de los principales consensos es que las interpretaciones del riesgo por los 
no especialistas se basan principalmente en sus propias creencias y convicciones y no en datos empíricos, lo cual es la base de la percepción de los técnicos y científicos (Slovic, 1987; Brown, 1992). Esto es crucial para entender cómo afectan los juicios de riesgo a la percepción del riesgo y, por lo tanto, para construir la base de cada iniciativa de comunicación de riesgos.

El análisis de los estudios de percepción y de comunicación de riesgo reforzó la hipótesis de que las experiencias previas son importantes en la percepción de riesgo de las personas (sobre todo entre los no especialistas) y, por tanto, decisivas para la comunicación de riesgos. Esto se evidencia en la literatura científica en este tema, donde la mayoría de los estudios llegaron a la conclusión de que el riesgo existe cuando se hace evidente o visible. De tal manera que cuando no hay ningún efecto visible, se tiene la impresión de que el riesgo es inexistente (Slovic, 1987; Moreno et al., 2010). Hallazgos similares se encontraron en un estudio pionero (Vaughan, 1993) sobre la percepción del riesgo y el comportamiento autoprotector asociado a la exposición crónica a plaguicidas.

Esto es crucial para entender cómo las iniciativas de comunicación de riesgos son (y serán) organizadas, además de ser fundamental para determinar la base en que se construirá cada iniciativa de comunicación de riesgos en la Región a partir de tres elementos fundamentales: confianza, credibilidad y experiencia (Peters et al., 1997).

Algunos estudios sobre la comunicación de riesgos entre poblaciones de migrantes latinos en los Estados Unidos de América han puesto de relieve que el verdadero peligro que enfrentan estas poblaciones dependerá de la forma en que perciben el riesgo y su respuesta. Por ejemplo, cuando se trata de conocer los peligros, entre las redes sociales de méxico-americanos (amigos, parientes, vecinos o compañeros de trabajo) se encuentran aquellos que tienen más credibilidad en cuanto a fuentes de información. Después de éstos vienen los medios de comunicación, los líderes de opinión y funcionarios del gobierno, estos últimos son los que tienen una baja credibilidad pública, como se ha visto, una condición fundamental para el desarrollo de la percepción del riesgo. En esta misma población minoritaria se identificó que, cuando se recibe un mensaje de advertencia, las personas tratan de verificar la información, rechazándola o ampliándola a través de la confirmación de diferentes fuentes (Lindell y Perry, 2004).

Las creencias basadas en la cultura pueden influir en las percepciones de la enfermedad, en la filtración de información de riesgo y en la tendencia a reaccionar ante las posibles amenazas a la salud (Moreno et al., 2010). Esto fortalece la presuposición de que la percepción del riesgo y la comunicación del riesgo son componentes indisociables del complejo escenario donde se llevan a cabo las relaciones de salud ambiental. Por lo tanto, y teniendo en cuenta algunas de los hallazgos que aquí se presentan, es necesario entender cómo la percepción del riesgo y comunicación del riesgo se articulan con el fin de construir un panorama de la comunicación de riesgos en América Latina.

\subsection{La comunicación de riesgos para el cuidado: una tendencia regional}

Hay tres aplicaciones principales (también conocidas como las "3 C" de la comunicación del riesgo): la comunicación de riesgos para la crisis, la comunicación de riesgos para el consenso 
y la comunicación de riesgos para el cuidado.

La comunicación desempeña un papel importante en la preparación, prevención, respuesta y recuperación de una emergencia o crisis de salud. Por lo general, este tipo de comunicación se asocia con el riesgo de emergencias, donde se necesita una respuesta inmediata, de lo contrario se pudiera poner en peligro la vida de las personas o grupos involucrados. En este tipo de comunicación, el riesgo de causar pánico con la información es menor que el daño causado por la falta de información; por consiguiente, no hay ningún requisito para asociar las acciones de comunicación con estudios/investigación destinados a apoyar una acción más completa. Esfuerzos en comunicación de riesgos se han visto en los sistemas de alerta temprana, a raíz del impacto tan importante que han tenido en los últimos años los huracanes y las lluvias intensas en muchos países de la región latinoamericana.

La comunicación de riesgos para el consenso se aplica para resolver conflictos entre los diferentes actores y grupos involucrados con el objeto de riesgo. También se echa mano de este enfoque en actores y grupos interesados en desarrollar un plan de acción para la gestión de riesgos. Algunos ejemplos incluyen consultas públicas sobre los rellenos sanitarios (y otros proyectos ambientales a gran escala), la instrumentación, las reuniones para la elaboración de los acuerdos de salud pública y la implementación de las iniciativas de gestión de riesgos. Este tipo de comunicación es donde menos experiencia se encuentra en América Latina, área que se tiene que reforzar de forma importante en particular cuando existe la necesidad de cierto tipo de instalaciones para la gestión del riesgo.

Por último, la comunicación de riesgos para el cuidado se basa en los resultados de investigación que tienen el objetivo de determinar las implicaciones de los riesgos para la salud y el medio ambiente. Este tipo de comunicación hace hincapié en la imposibilidad de separar la investigación de la acción; siendo además una herramienta fundamental para hacer frente a los problemas planteados durante una evaluación de riesgos o proyecto de investigación y deben asociarse con el entendimiento de cómo las personas responden ante una situación de riesgo o una amenaza inminente.

Es importante destacar que cuando se utiliza la comunicación de riesgos para el cuidado -con el objetivo de cambiar el comportamiento individual o colectivo- es necesario para salvar estas iniciativas apoyarse en estudios culturales, en particular con enfoques de percepción de riesgos, ejemplos latinoamericanos se observan en la tabla 4. Como se ha visto, se puede aproximar a las creencias, temores y sentimientos con el objeto de la comunicación, calibrando la respuesta y haciendo la comunicación de riesgos más efectiva (figura 3 ). Al considerar que no es posible disociar los cambios de actitud y la subjetividad individual/colectiva, también es imposible disociar, en estos casos, la percepción del riesgo y su comunicación.

Lo anterior refuerza los argumentos para la incorporación de los estudios de percepción del riesgo en las iniciativas de comunicación de riesgos, sobre todo aquellas para el cuidado. Sólo entonces vamos a ser capaces de explicar cómo la forma de concebir el mundo de los "especialistas" y los "no especialistas" se origina, y se entiende entonces el significado de cada peligro que enfrentan, lo que debería ser el punto de partida para todas y cada una de las iniciativas de comunicación de riesgos. 
Figura 3. Esquema de una comunicación de riesgos para el plan de atención

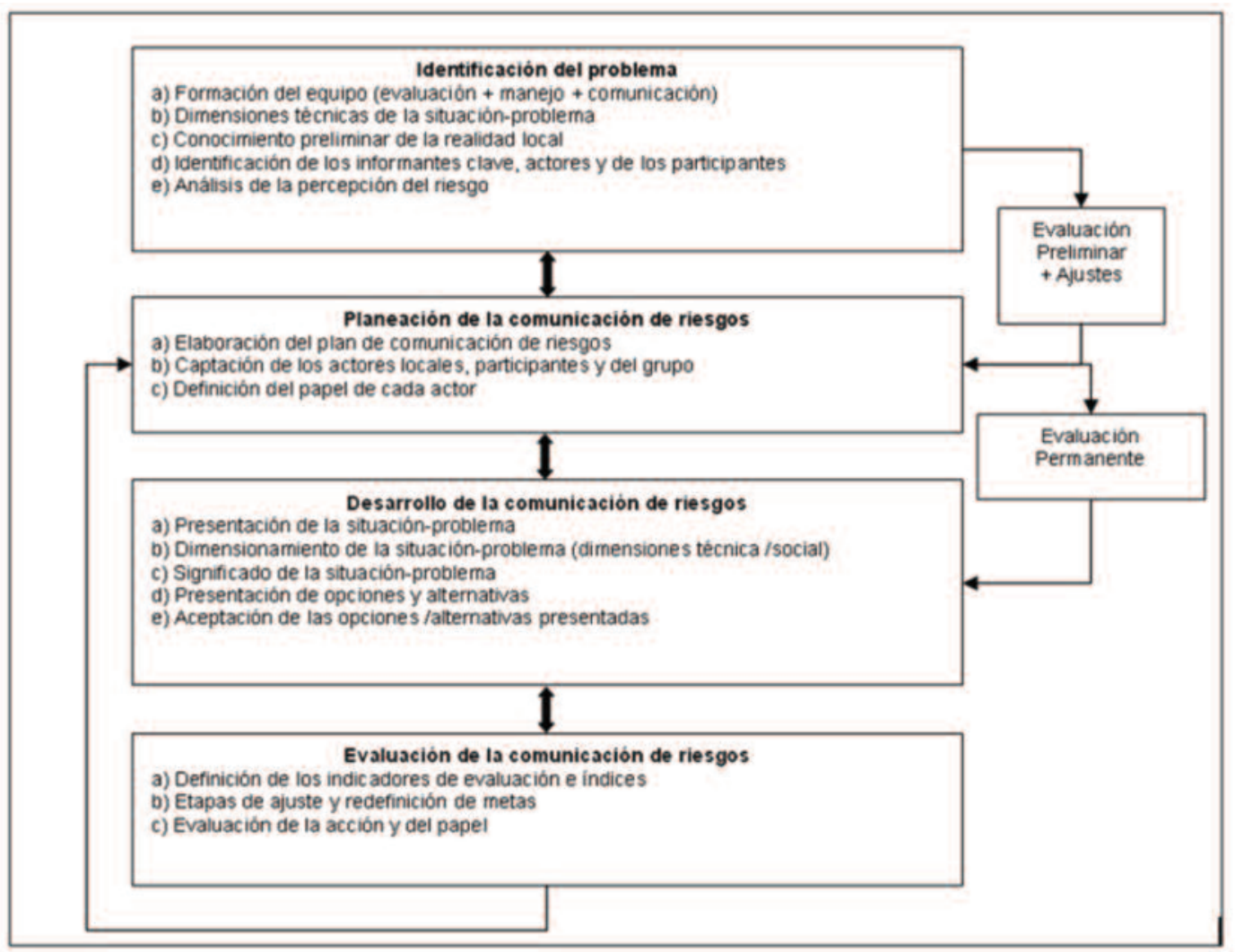

Fuente: elaboración propia

\section{Conclusiones}

Uno de los elementos para poder disminuir la carga de enfermedades asociadas al medio ambiente es tener claro aquellos determinantes y factores de riesgo que se pueden identificar en las principales poblaciones vulnerables. Para ello, se debe contar con el fortalecimiento de la investigación y con la vigilancia epidemiológica ambiental en donde el monitoreo ambiental juega un papel muy importante. Lo anterior proporcionará la plataforma para el establecimiento de políticas públicas para la protección de la salud, así como para el fortalecimiento de alianzas estratégicas intersectoriales e interinstitucionales, a fin de asegurar la efectividad de las intervenciones (OPS, 2007).

Otro elemento que propicia el éxito de las políticas públicas son los cambios de conducta que deben ser sostenidos y permanentes junto con el mejoramiento de la calidad del medio ambiente, además de los institucionales y de políticas que conduzcan a que la ciudadanía pueda realmente optar por tener una vida en un ambiente sano y estable, en donde los servicios am- 
bientales no se vean amenazados. Para ellos, se requiere un trabajo en colaboración cercana con la industria, los medios de comunicación, sociedad civil, el sector académico y otros actores estratégicos, relación basada en un compromiso social (OPS, 2007). En todo lo anterior, la comunicación de riesgos juega un papel fundamental.

\section{Referencias}

Brown, Phil. (1992). Popular epidemiology and toxic waste contamination: lay and professionals way of knowing. Journal of Health and Social Behavior. Vol. 33, n 3, pp. 267-281.

Catalán-Vázquez, Minerva; Schilmann, Astrid y Riojas-Rodríguez, Horacio. (2010). Perceived health risks of Manganese in the Molango mining District, Mexico. Risk Analysis. Vol. $30 n^{\circ} 4$, pp. 619-634.

Kleinman, Arthur. (1980). Patients and healers in the context of cultures: An exploration of borderland between Anthropology and Psychiatry. Berkeley: University of California Press.

Kleinman, Arthur y Good, Byron. (1986). Culture and depression: Studies in anthropology \& cross-cultural psychiatry of affect \& disorder. Berkeley: University of California Press.

Lindell, Michael y Perry, Ronald. (2004). Communicating Environmental Risk in Multiethnic Communities. Thousand Oaks: Sage Publications.

Lundren, Regina y McMakin, Andrea. (2004). Risk communication. A Handbook for communicating environmental, safety, and health risks. Batelle Press, Columbus, Ohio: Library of the Congress.

Moreno, Ana Rosa; Cubillas, Ana Cristina; Guerra García, Alberto y Peres, Frederico. (2010). La comunicación de riesgos en América Latina. En Galvão, Luiz Augusto; Finkelman, Jacobo y Henao, Samuel (Eds.) Los determinantes ambientales y sociales de la salud. Washington, D.C.: OPS.

Organización Panamericana de la Salud. (2007). Agenda para la Salud en las Américas 20082017. Washington, D.C.: OPS:

Rozemberg, Brani y Peres, Frederico. (2003). Reflexões sobre a educação relacionada aos agrotóxicos em comunidades rurais. En Peres, Frederico y Moreira, Josino Costa (Orgs.). É veneno ou é remédio? Agrotóxicos, saúde e ambiente (pp. 367-384). Rio de Janeiro: Fiocruz.

Peters, Richard; Covello, Vincent y McCallum, David. (1997). The determinants of trust and credibility in environmental risk communication: an empirical study. Risk Analysis. Vol. 17, pp. 43-54.

Prüss-Üstün, Annette y Corvalán, Carlos. (2006). Ambientes saludables y prevención de enfermedades. Hacia una estimación de la carga de morbilidad atribuible al medio ambiente. OMS. Disponible en http://www.who.int/quantifying_ehimpacts/publications/prevdisexecsumsp.pdf. Recuperado el 24 de septiembre de 2010.

Sjöberg, Lennart y Fromm, Jana. (2001). Information technology risks as seen by the public. Risk Analysis. Vol. 21, n³, pp. 427-441.

Slovic, Paul (1987). Perception of risk. Science. Vol. 236, pp. 280-285. 
Vaughan, Elaine. (1993). Chronic Exposure to an Environmental Hazard: Risk Perceptions and Self-Protective Behavior. Health Psychology. Vol. 12, n 1, pp. 74-85.

Wiedemann, Peter (1993) Introduction risk perception and risk communication. Jülich: Programme Group Humans; Environment, Technology (MUT), Research Centre Jülich. (Arbeiten zur Risko-Kommunikation 38 - mimeo).

Zola, Irving (1973). Pathways to the doctor: from person to patient. Social Sciences and Medicine. Vol. 7, pp. 677-89. 